\title{
Interpretation of Variants of Uncertain Significance in the Clinical Setting: A Case of Treatable Ataxia
}

Miguel Wilken ( $\nabla$ mwilken@fleni.org.ar)

Malco Rossi

Raul Carrea Institute for Neurological Research (FLENI) https://orcid.org/0000-0003-3383-8566

Marcelo Merello

Fundacion para la Lucha contra las Enfermedades Neurologicas de la Infancia

\section{Case report}

Keywords: Spinocerebellar ataxia type 38 (SCA38), genetic, Variants of Uncertain Significance, ELOVL5, docosahexaenoic acid (DHA)

Posted Date: August 10th, 2020

DOI: https://doi.org/10.21203/rs.3.rs-55670/v1

License: (c) (i) This work is licensed under a Creative Commons Attribution 4.0 International License. Read Full License 


\section{Abstract}

Background: Spinocerebellar ataxia type 38 (SCA38) is an autosomal dominant cerebellar ataxia caused by pathogenic variants in the elongation of very long chain fatty acids-like 5 gene (ELOVL5). Improvement of ataxia with a docosahexaenoic acid (DHA) replacement therapy has been reported.

Case presentation: A 73-year-old man of Hispanic descent presented with gait and limb ataxia, dysarthria, slow and hypometric saccades, hearing loss, mild cognitive impairment, and hypopalesthesia. The initial scale for the assessment and rating of ataxia (SARA) score was 11. After a negative routine workout for ataxia and testing for common forms due to expanded repeats, whole-exome sequencing (WES) identified a heterozygous variant $(c .327+1 \mathrm{G}>A)$ in the ELOVL5 gene that was predicted to have a negative effect on splicing but was categorized as a Variant of Uncertain Significance (VUS). The patient was started on DHA $600 \mathrm{mg} /$ day. Four months later, the patient showed a considerable reduction in the scale for the assessment and rating of ataxia (SARA) score, from 11 to 5 points, with a clear improvement in gait and limb ataxia that was sustained at 24 months of follow-up.

Conclusions: We illustrate the case of a patient presenting with a variant considered genetically and biochemically of uncertain significance. Despite being a VUS, its location in a gene that is known to cause ataxia (SCA38), as well as a compatible phenotype, led to the interpretation of this variant as probably pathogenic from a clinical practice standpoint, especially considering prior reports that showed clinical improvement with a specific, over-the-counter, pharmacological treatment. A further satisfactory response to treatment supported our clinical approach.

\section{Background}

Next-generation sequencing technologies have been increasingly used in recent years in clinical practice. These studies usually yield an enormous amount of data with genetic variants, whose correct interpretation remains challenging. Variants are commonly deemed pathogenic or benign according to type or location of the mutation, family history, functional studies, prevalence in affected and healthy individuals, and previously published data. However, if the variant does not fulfill criteria for pathogenicity or benignity, or if the supporting data is conflicting, it is considered of uncertain significance (VUS) [1]. Interpretation of such variants is especially difficult in the clinical setting, as there is no guideline or standard for the adequate rendering of these results.

We present a patient with ataxia in which a VUS was obtained in a gene that is known to cause ataxia and for which a specific treatment is available.

\section{Case Presentation}

A 73-year-old man consulted our Movement Disorders clinic complaining of dizziness that started 4 years earlier and progressive unsteady gait with frequent falls for 1.5 years before consultation. On physical examination, he presented mild bilateral hearing loss, distal hypopalesthesia, and moderate gait ataxia. 
He also presented mild dysarthria, and his finger chase, heel-shin slide and fast alternating movements were also affected. The initial scale for the assessment and rating of ataxia (SARA) score was 11. No pyramidal, parkinsonian or dystonic features were observed. He presented slow and hypometric saccades. Cognitive evaluation showed mild executive, attention and working memory impairment. His family is of Hispanic descent, with no prior history of ataxia.

A brain MRI showed generalized cortical and mild cerebellar atrophy as well as multiple and confluent hyperintense lesions on T2 and FLAIR sequences. Bilateral globus pallidus hyperintensities on GRE were observed, which were later on categorized as mild calcifications on brain CT.

After a negative routine workout for ataxia and testing for common forms due to expanded repeats, whole-exome sequencing (WES) identified a heterozygous variant (c.327+1G>A) in the ELOVL5 gene that was predicted to have a negative effect on splicing, but was categorized as a VUS. The ELOVL5 gene codifies an elongase involved in polyunsaturated fatty acid synthesis. Pathogenic mutations in this gene generate a docosahexaenoic acid (DHA) and arachidonic acid deficit, which causes spinoerebellar ataxia type SCA38 (ATX-ELOVL5) [2]. Moreover, there are reports showing improvement of ataxia with DHA replacement therapy $[3,4]$. Even though the patient harbored a putative VUS, he was started on DHA 600 $\mathrm{mg} /$ day, as previously reported $[3,4]$. Four months later, the patient showed a considerable reduction in SARA score, from 11 to 5 points, with a clear improvement in gait and limb ataxia sustained at 12 and 24 months (Table 1).

\section{Discussion And Conclusions}

We illustrate the case of a patient presenting with a variant considered genetically and biochemically of uncertain significance. Despite being an unreported variant, its location in a gene that is known to cause ataxia (SCA38), as well as a compatible phenotype, led to the interpretation of this variant as probably pathogenic from a clinical practice standpoint, considering prior reports that showed clinical improvement with a specific, over-the-counter, pharmacological treatment. A further satisfactory response to treatment supported our clinical approach.

Spinocerebellar ataxias are a group of clinically and genetically hetegeneous diseases characterized by ataxia in association with several other non-cerebellar clinical manifestations [5]. Spinoerebellar ataxia type SCA38 (ATX-ELOVL5) is characterized by gait and limb ataxia, associated mainly with hearing loss, neuropathy, nystagmus and ophthalmoparesis [2]. In a double-blind randomized placebo-controlled trial, followed by an open-label study with overall 40-week of treatment, DHA supplementation was found to be safe and clinically effective in ten SCA38 (ATX-ELOVL5) patients [3]. Improvement in cerebellar features was also corroborated by significant improvement of cerebellar hypometabolism [3]. The favorable response to prolonged DHA supplementation in the patient here reported is in line with other reports $[4,6]$.

The individual interpretation of genetic sequencing datasets is not clear cut. Current efforts in developing a systematic approach have focused on biochemical pathogenicity [7]. However; clinical clues are always 
mandatory for adequate interpretation of ancillary test results and define the clinical significance of VUS, especially for treatable conditions.

\section{Abbreviations}

SCA38: Spinocerebellar ataxia type 38

ELOVL5: elongation of very long chain fatty acids-like 5 gene

DHA: docosahexaenoic acid

SARA: scale for the assessment and rating of ataxia

WES: whole-exome sequencing

VUS: Variant of Uncertain Significance

\section{Declarations}

Ethics approval and consent to participate: This study was approved by the Institutional Review Board of FLENI. The patient provided written informed consent for participation in this study.

Consent for publication: the patient provided written informed consent for publication.

Availability of data and materials: The datasets used and/or analyzed during the current study are available from the corresponding author on reasonable request.

Competing interests: The authors declare that they have no competing interests.

Funding: None to declare.

Authors' contributions: MW, MR, and MM contributed to the conception and design of the study. MW and MR were responsible for collection of the clinical data. MW, MR and MM analyzed genetic data obtained from the WES performed in a prestigious and worldwide leader European laboratory dedicated to the highest quality genetic testing.

MW and MR wrote the manuscript and all authors were responsible for its review and critique. All authors read and approved the final manuscript.

Acknowledgements: We thank the patient for their willingness to participate in this study.

\section{Authors' information}

${ }^{1}$ Movement Disorders, Neurology Department, FLENI. Montañeses 2325 (C1428AQK), 
Buenos Aires, Argentina.

${ }^{2}$ Clinical Neurophysiology, Neurology Department, FLENI. Montañeses 2325

(C1428AQK), Buenos Aires, Argentina.

${ }^{3}$ Argentine National Scientific and Technological Research Council (CONICET). Godoy

Cruz 2290 (C1425FQB), Buenos Aires, Argentina.

${ }^{4}$ Pontificia Universidad Católica Argentina (UCA). Av. Alicia Moreau de Justo 1600, (C1107AFF), Buenos Aires, Argentina.

\section{References}

1. Richards S, Aziz N, Bale S, et al. Standards and Guidelines for the Interpretation of Sequence Variants: A Joint Consensus Recommendation of the American College of Medical Genetics and Genomics and the Association for Molecular Pathology. Genet Med. 2015;17:405-424.

2. Di Gregorio E, Borroni B, Giorgio E, et al. ELOVL5 mutations cause spinocerebellar ataxia 38. Am J Hum Genet. 2014;95:209-217.

3. Manes M, Alberici A, Di Gregorio E, et al. Docosahexaenoic acid is a beneficial replacement treatment for spinocerebellar ataxia 38. Ann Neurol. 2017;82:615-621.

4. Manes M, Alberici A, Di Gregorio E, et al. Long-term efficacy of docosahexaenoic acid (DHA) for Spinocerebellar Ataxia 38 (SCA38) treatment: An open label extension study. Parkinsonism Relat Disord. 2019;63:191-194.

5. Rossi M, Perez-Lloret S, Cerquetti D, Merello M. Movement disorders in autosomal dominant cerebellar ataxias: A systematic review. Mov Disord Clin Pract. 2014;6:154-160.

6. Gazulla J, Orduna-Hospital E, Benavente I, et al. Contributions to the study of spinocerebellar ataxia type 38 (SCA38). J Neurol. 2020;267:2288-2295.

7. Trinh J, Tadic V, Klein C. How do I confirm that a new mutation is pathogenic? Mov Disord Clin Pract. 2018;5:229.

\section{Tables}

Table 1. SARA scores before and after treatment with docosahexaenoic acid. 


\begin{tabular}{|lllll|}
\hline SARA & Baseline & 4 months & 12 months & 24 months \\
\hline 1 - Gait & 2 & 1 & 1 & 1 \\
\hline 2 - Stance & 3 & 3 & 2 & 2 \\
\hline 3 - Sitting & 0 & 0 & 0 & 0 \\
\hline 4 - Speech disturbance & 1 & 0 & 0 & 0 \\
\hline 5 - Finger chase & 1 & 1 & 0 & 0 \\
\hline 6 - Nose-finger test & 0 & 0 & 0 & 0 \\
\hline 7 - Fast alternating hand movements & 2 & 0 & 1 & 1 \\
\hline 8 - Heel-shin slide & 2 & 0 & 0 & 0 \\
\hline TOTAL & 11 & 5 & 4 & 4 \\
\hline
\end{tabular}

SARA: Scale for the assessment and rating of ataxia 